\title{
TraffCon: An Intelligent Traffic Control System for Wireless Vehicular Networks
}

\author{
Kevin Collins ${ }^{1}$, Gabriel-Miro Muntean 1 \\ 1 Performance Engineering Laboratory, RINCE, \\ School of Electronic Engineering, Dublin City University, Dublin, Ireland \\ \{kevin.collins, munteang\}@eeng.dcu.ie
}

\begin{abstract}
Traffic Congestion is a very serious problem which is becoming ever worse as the growth in the number of cars on the road significantly outpaces the provision of road capacity. This paper presents TraffCon, a novel Traffic Management System (TMS) for Wireless Vehicular Networks that combats this problem by seeking to optimize the usage of the existing road capacity. It also outlines an architecture which includes a novel server-side decision making module, that enables the dissemination of instructions to vehicles; if followed these result in optimal road usage.
\end{abstract}

Keywords: Traffic Management, Road Vehicles, Driver Instruction, Wireless Networks

\section{Introduction}

Wireless Access in Vehicular Environments (WAVE) has been an active research area for some time. WAVE is still in the standardization phase and several groups are working to that end, such as the Car 2 Car Communication Consortium [1], the Dedicated Short Range Communications working group [2] and the IEEE 802.11p task group [3]. Regardless of the outcome of this process the potential for the provision of new applications in the vehicular realm is evident, be they Internet based, convenience, safety or traffic management applications.

Much of the early research into viable use-cases for WAVE has focused on providing a safety benefit. The Vehicle Safety Communications Consortium has created a long list of distinct use-cases ranked according to their benefits [4]. The majority but not all of these are safety related. At present many projects worldwide are developing safety applications based on these or similar scenarios [5, 6, 7].

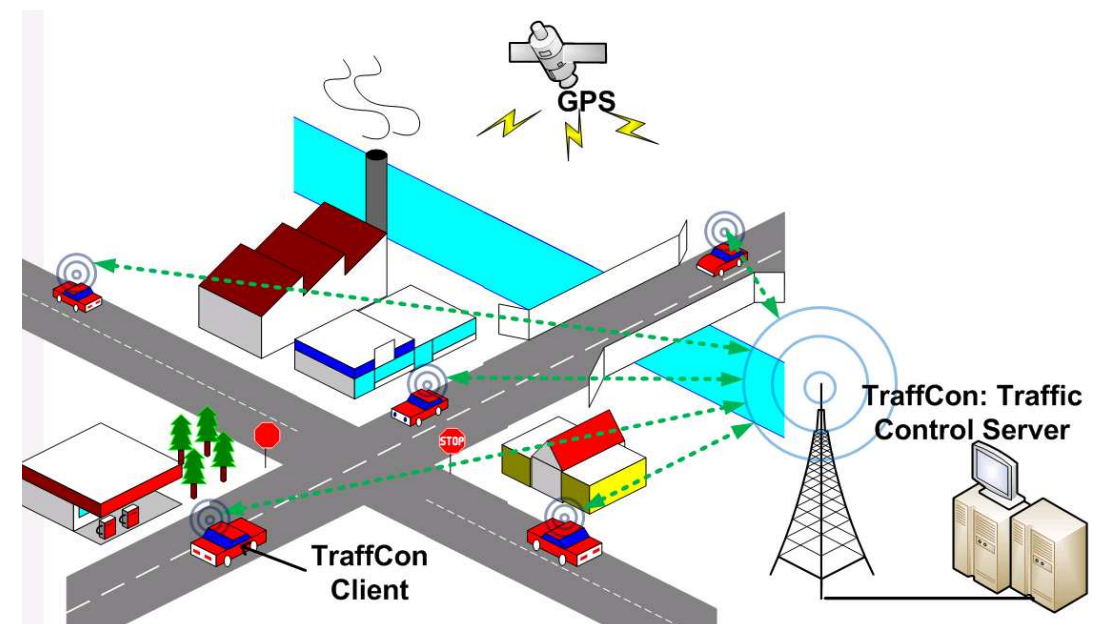

Figure 1: Traffic Management System (TMS) for WAVE 
More recently academics have begun to branch out into different research directions involving WAVE. Some applications under development include: MobEyes: a proposal to use vehicles as sensors in a mobile surveillance platform for urban monitoring [8], FleaNet: a virtual "flea market" allowing drivers and roadside shops to advertise their demands/offers [9] and free parking space discovery [10].

One use case for WAVE which has enormous potential is Traffic Management. Vehicular traffic is one of the great ills of modern society; in urban areas commuters can spend a significant percentage of their life stuck in traffic. It has been estimated by the Texas Traffic Institute that traffic congestion will cost the US over \$90bn per year by 2009 [11] and the UK Treasury put the cost to its country's economy at $£ 20 \mathrm{bn}$ (US $\$ 38 \mathrm{bn}$ ) for 2006 [12]. These are huge monetary costs, based on lost productivity and wasted fuel but there is also the environmental cost to consider. Disturbingly the situation is set to worsen as the number of vehicles on the road outpaces growth in road capacity worldwide. From 1982 to 2002, the number of vehicles in the US grew by $36 \%$ and vehicle miles travelled by $72 \%$ while road capacity increased by less than 5\%. Between 1990 and 2004 the number of cars in the $25 \mathrm{EU}$ member states rose by over $40 \%$ and continues to rise, from 1990-1998 total length of motorways in the EU grew by $28 \%$ but has remained roughly stagnant since then $[11,12]$.

This paper proposes TraffCon: a novel Traffic Management System (TMS) for WAVE (figure 1). The main aim of TraffCon is to improve the overall Quality of Driving Experience (QDE). To achieve this objective the overriding emphasis of such a system is to reduce traffic congestion/increase traffic flow, by maximising the usage of the available infrastructure. TraffCon's benefits are varied: social, economic and environmental i.e. shorter journey times, financial savings, increased productivity and a reduction in vehicle emissions.

The rest of this paper presents the proposed TMS and is organized as follows. Section 2 discusses related works, section 3 deals in greater detail with the TMS concept, section 4 outlines the proposed TraffCon-based solution, section 5 describes how TraffCon will be tested and conclusions are drawn in section 6.

\section{Related Works}

There are many research groups exploring use cases for WAVE which improve QDE by influencing traffic conditions. These can be loosely divided into three main categories Traffic Information/Advisory Systems (TIS), Autonomous Vehicle Systems and Traffic Management Systems.

\subsection{Traffic Information/Advisory Systems (TIS)}

A number of TISs have been developed i.e. systems which gather traffic data and disseminate traffic information to users, so they can make better informed decisions regarding their route. Examples of this include TrafficView: a device which gives drivers an extended horizon i.e. a real time view of road traffic far beyond what they can actually see [13], StreetSmart: a system which identifies and disseminates traffic patterns to users [14] and SOTIS: a system which distributes up-to-date travel and traffic information pertinent to a vehicles locale [15].

While these systems do keep drivers better informed about traffic conditions, there is no telling how the driver will interpret the information given. Consequently there is no guarantee such systems lead to more beneficial or optimal route decisions. Much work has been also done exclusively in the area of Data Harvesting and Information Dissemination schemes for WAVE [15, 16, 17]

\subsection{Autonomous Vehicle Systems}

Autonomous vehicle systems can provide traffic control solutions by fully automating vehicles and thereby removing user responsibility for driving. There has been and continues to be a wealth of research in this area, the most celebrated of which feeds into the DARPA Grand Challenge [18]. Some notable recent work includes a vehicle capable of navigating complex environments using artificial vision [19], a perception and planning architecture for autonomous vehicles [20] and a system capable of 
avoiding complex obstacle filled environments to complete a journey described by a simple set of waypoints [21]. However at present such solutions are prohibitively expensive for large scale deployment and must also overcome the challenge of user resistance to automation.

\subsection{Traffic Management Systems (TMS)}

Systems which actively control aspects of the traffic network in order to force member nodes into a behaviour which has some benefit to the system as a whole can be classified as TMS's. Current work in the area includes adaptive traffic lights for improved traffic co-ordination at intersections [22] and train vehicle communications to manage their interactions at road and rail intersections [23].

\section{Traffic Management System (TMS)}

There are enormous challenges in developing a fully functional large scale TMS, i.e. for a large urban area. Even for a modest sized urban area such as the town of Cambridge (UK) population 100,000, there are 183,850 vehicles passing through it in the 12 hours from 07:00 to 19:00 on a typical day [24]. When a large metropolitan area is considered it is clear that harvesting traffic data from vehicles will yield vast volumes of data. Storing this data may prove problematic not to mention processing it in realtime and disseminating control messages.

However the first step in the development is to determine - What can potentially be changed/controlled in order to alter/manage traffic conditions?

- A vehicles route - Vehicles may be directed to follow a specific path en route to their destination.

- A vehicles lane - Vehicles may be directed to change their lane e.g. bus lanes could potentially be used to increase capacity provided buses are not delayed.

- Vehicle speed - Vehicles may be instructed to adjust their speed.

- Traffic light interval times - The green light times and ratios (i.e. favour one road over another at a junction) may be adjusted.

A simple indicator of traffic congestion is the ratio of the number of vehicles on the road to road capacity. Re-routing and adjusting lane positions of vehicles allows the road capacity to be maximized thereby reducing congestion and increasing the flow of traffic. Spaces between vehicles occupy road capacity in the same way vehicles do. If vehicle speed can be controlled to minimize spaces between vehicles, then road capacity can be further maximized. When traffic lights are red they disrupt the flow of traffic; optimizing traffic light operation to make traffic flow as arterially as possible is clearly beneficial.

While manipulating these elements of the traffic system can improve traffic flow other factors must not be neglected in the quest for speed. The stress of sitting in traffic should not be replaced by an irritating or overly invasive interface directing drivers in the cockpit, the system should not force drivers to drive in a manner which is erratic or uncomfortable and it should in no way endanger the safety of the driver. In short for any TMS safety is paramount, reducing journey time is vital, but comfort is important too.

In the future there may be other infrastructural elements a TMS could interact with e.g. future roads may be designed so that their layout can be altered (i.e. painted white lines are replaced by some form of electronic display which can be modified).

\section{TraffCon: Intelligent Traffic Control Solution}

This paper focuses solely on managing vehicle routes. It is assumed that vehicles have a GPS receiver connected to a computing device with wireless connectivity. 


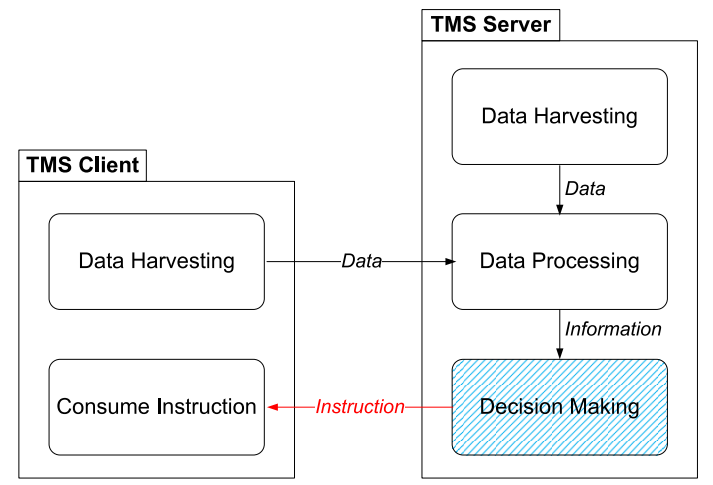

Figure 2: TMS Architecture with Server Side Decision Making for Instruction Dissemination

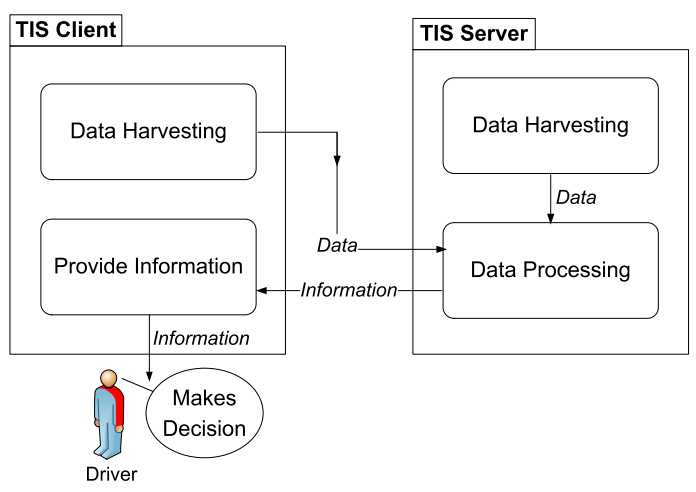

Figure 3: Traditional TIS Architecture

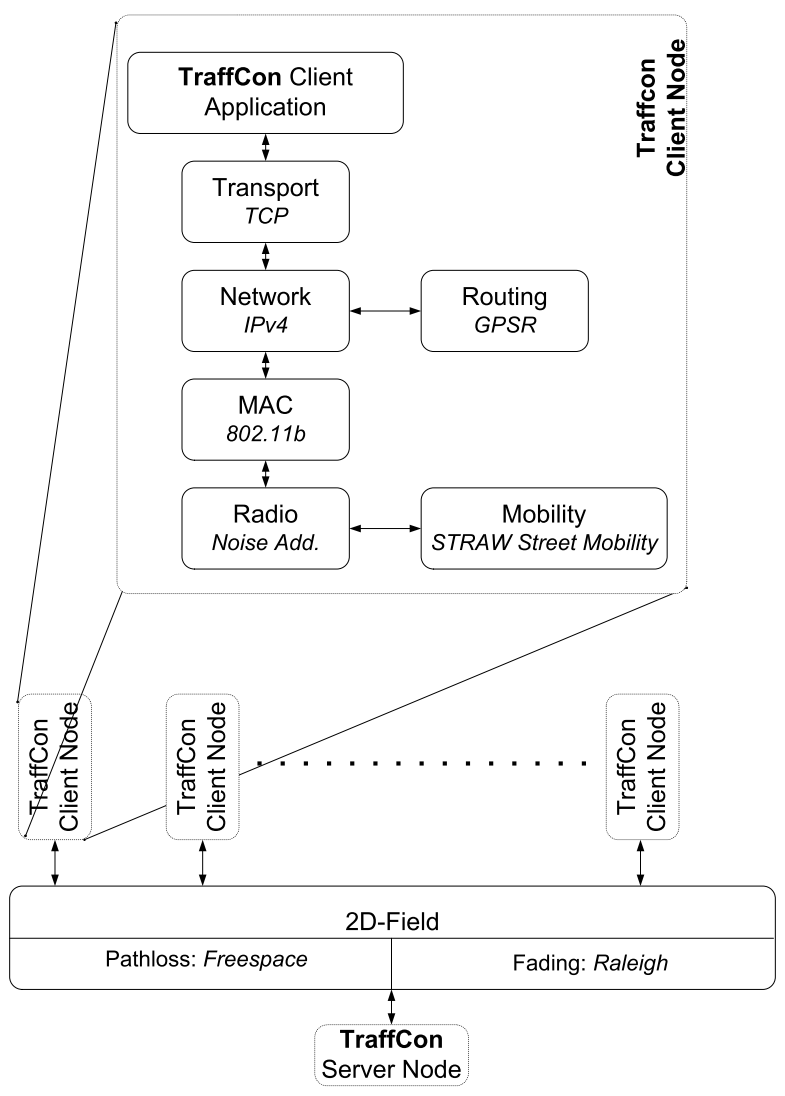

Figure 4: A Possible TraffCon Configuration for JiST/SWANS-STRAW Simulation

\subsection{System Architecture}

TraffCon has a client server architecture. Vehicles / client nodes communicate with server nodes responsible for traffic management. The systems functional blocks are divided between client and sever as shown in figure 2. Server side decision making means instructions are disseminated to clients. This architecture is what differentiates this TMS from traditional Traffic Information/Advisory Systems (TIS) where information is disseminated to the clients and drivers are responsible for decision making as seen in figure 3.

\subsection{System Functional Blocks}

The system is comprised of four main functional blocks:

1. Data Harvesting - all nodes in the system gather useful traffic data.

2. Data Processing - the data is filtered, aggregated and refined to generate precise information regarding the state of the traffic network.

3. Decision Making - the traffic network information is used in a decision making process which generates a route instruction which if followed has a benefit over the other route choices available e.g. improved traffic flow, a reduction in fuel consumption

4. Instruction Consumption - the instruction is consumed i.e. it is followed or ignored. 


\subsubsection{Data Harvesting}

All vehicles in the TraffCon system will gather data regarding the state of the road network. In order to understand the data collected, it is necessary to define two components which can be used to describe the road network-

- Junction: Point where two or more roads meet

- Link: Section of road between two junctions.

For two junctions $\mathrm{J}$ and $\mathrm{K}$ joined by a single section of road, there are two links connecting them i.e. the link JK which allows traffic to travel from $\mathrm{J}$ to $\mathrm{K}$ and the link $\mathrm{KJ}$ which allows traffic to flow from $\mathrm{K}$ to $\mathrm{J}$.

It is assumed all vehicles carry map data for the area in which they travel. At the most basic level this data is simply the GPS co-ordinates of all junctions in the area. Given that all nodes know their own location (from their GPS receiver) and the locations of all junctions in the area, then a node can identify when it has reached a junction.

Initially two pieces of data are obtained from a vehicle before it begins its journey i.e. starting location and destination location. While in transit a vehicle constantly checks whether it is at a junction; if a junction is reached a timestamp is set. Whenever a vehicle has traversed a link the time taken to do so (i.e. the link time), is calculated. This link time, the time the vehicle entered the link (reached the first junction) and the link ID (two GPS co-ordinates i.e. longitude-latitude pairs; $\mathrm{J}$ and $\mathrm{K}$ for the junctions at the beginning and end of the link combined in the form JK) are packaged and sent to the server. Table 1 shows the data format a small sample set of data passed by clients to a server.

Table 1: Data harvested by nodes

\begin{tabular}{cccc}
\hline \hline Node ID & Link ID & Link Start Time & Link Time $(\mathrm{ms})$ \\
\hline 1 & $-71.040778+42.285664-71.040694+42.285698$ & $2007-05-1107: 57: 46$ & 13000 \\
2 & $-71.040778+42.285664-71.040694+42.285698$ & $2007-05-1107: 58: 33$ & 12800 \\
3 & $-71.040778+42.285664-71.040694+42.285698$ & $2007-05-1107: 58: 53$ & 13000 \\
\hline
\end{tabular}

By gathering such simple data the server can generate a wide range of useful information such as; average link times, average link speeds, instantaneous per link vehicle density, etc.

\subsubsection{Data Processing}

The data set described above is used to generate a table of average link times in the format shown in table 2 by using window-based averaging. Given that the server also knows the length of every link then a table of average link speeds is also generated using speed $=$ distance/time .

Table 2: Window-based Average link times for data from Table 1

\begin{tabular}{cc}
\hline \hline Link ID & Average Link Time $(\mathrm{ms})$ \\
\hline$-71.040778+42.285664-71.040694+42.285698$ & 12960 \\
\hline
\end{tabular}

\subsubsection{Decision Making}

In a TIS the user is responsible for making route decisions. They are given realtime traffic information and it is assumed they can make route adjustments that are beneficial to themselves. As a result the system is tailored to only benefit individual client nodes. The emphasis is solely on getting individuals to their destination as quickly as possible, with no consideration for the effect on the overall traffic system.

For the TMS with server side decision making, the overall situation is of paramount importance and vehicles are given route instructions designed to benefit both the individual and the overall system. 


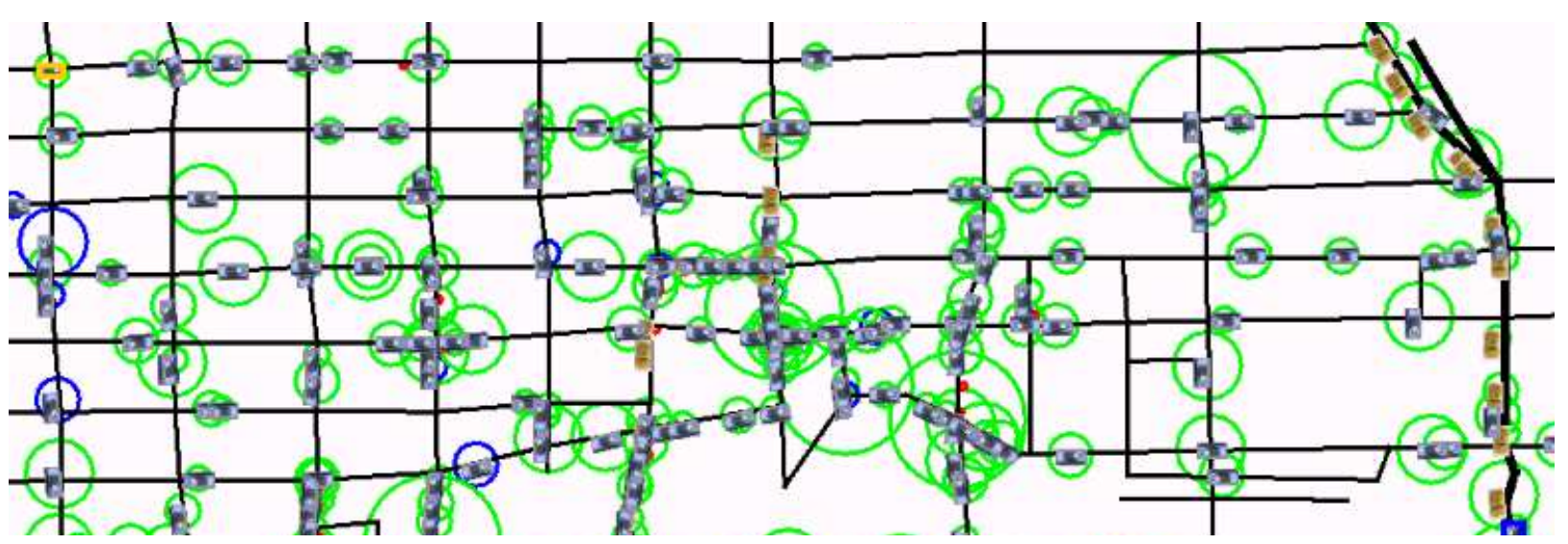

Figure 5: Simulator Screengrab: Vehicles move in a real world city and send and receive packets (indicated by flashing circles)

Genetic Algorithms are used as they are a suitable approach for solving such a combinatorial optimization problem [25]. The fitness function eq. 1 is proposed to make route decisions which minimize journey time and fuel consumption. Parameters for overall and individual benefit are used.

$$
F(y)=w_{1} / J(y)+w_{2} / I(y)+w_{3} / E(y)+w_{4} / D(y)+w_{5} S(y)
$$

Where; $\mathrm{J}(\mathrm{y})$ is average journey time in the system, $\mathrm{I}(\mathrm{y})$ is individual node's journey time, $\mathrm{E}(\mathrm{y})$ is average fuel consumption in the system, $\mathrm{D}(\mathrm{y})$ is individual node's fuel consumption, $\mathrm{S}(\mathrm{y})$ is solution fairness (designed to keep a balance between the benefit to the individual and to the system as a whole such that no individual is overly rewarded/penalised) and $w_{i}$ are weighting factors.

The information made available in the data processing stage is pulled as required to evaluate parameters. For Example trip times can be estimated by summing the average link times along a route. It is possible to enhance this function at a later date by considering additional parameters e.g. speed and/or jitter.

\subsubsection{Instruction Consumption}

An interface of some kind is required in TraffCon enabled vehicles to present instructions to the driver for consumption. Options include an audio solution or a visual solution such as Head-Up Display (HUD). Regardless of interface drivers should receive pertinent instructions in a timely fashion.

\section{Testing}

In order to evaluate the proposed solution the Java in Simulation Time / Scalable Wireless Ad hoc Network Simulator (JiST/SWANS) is used in conjunction with the Street Random Waypoint (STRAW) vehicular mobility model for network simulations [26, 27, 28]. The vehicular mobility model employed by the simulator uses real world road maps as seen in figure 5 . This simulator setup allows the wireless network and vehicular mobility aspects of the system to be simulated simultaneously.

The existing model was enhanced with data harvesting and data processing modules which follow the descriptions in sections 4.2.1 and 4.2.2 respectively. No decision making module has been deployed as yet. It is planned to model the consumption of instructions with varying percentages of obedience.

A potential simulation configuration is shown in figure 4; client nodes move in a field according to STRAW Street Mobility and run the TraffCon Client Application, TCP is used at the transport layer, IPv4 at the network (in conjunction with Greedy Perimeter Stateless Routing (GPSR) [29] ) and 802.11b at the MAC, in this setup. There is a single sever node which runs the TraffCon Server Application, and is static rather than mobile but is otherwise identically configured. 
In order to evaluate system performance the behaviour of TraffCon will be contrasted against two other benchmark systems, across a range of parameters: average node - speed, journey time, fuel economy, jitter etc.

The testbed will include:

1. A model where all vehicles attempt to take the shortest route to their destination (in spatial terms).

2. A model where vehicles run a TIS which instructs them to take the quickest route, by using; Dijkstra's Algorithm to make route decisions based on average link time information supplied by the server.

3. A model where vehicles run the proposed TraffCon-based system.

The first system models the real world behaviour of vehicles with no navagational aids. The second examines what happens when vehicles are routed optimally but in a greedy fashion i.e. with no regard for the effect on other vehicles. The third will show the result of routing vehicles optimally with concern for the overall system. In both the second and third cases the effect of varying the penetration rate of the technology will also be examined.

\section{Conclusion}

Traffic congestion is already a major problem worldwide and it is becoming more and more serious because the number of cars on the road is increasing at a higher rate than road capacity. In this context this paper has introduced TraffCon a novel traffic management system for WAVE, which aims to optimize the usage of existing road capacity. The system architecture has been fully outlined and three main functional blocks Data Harvesting, Data Processing and Decision Making have also been described in the detail.

With regard to testing, the Data Harvesting and Processing functionality has been implemented on the JiST/SWANS-STRAW simulator and a full system performance evaluation procedure has been detailed.

Future work will involve full implementation and evaluation of the TraffCon system. Further enhancements are envisaged to include the addition of a feedback loop to attribute congestion charges/credits to drivers based on whether they disobey/comply with instructions received. Such a penalty/reward paradigm adds greater likelihood of compliance and brings the system closer to true control. Vehicle lane and traffic signal control may also be added. These enhancements would help to further maximize road capacity.

\section{Acknowledgements}

The support of the Irish Research Council for Science, Engineering and Technology Embark Initiative is gratefully acknowledged.

\section{References}

[1] “Car 2 Car Communication Consortium.” http://www.car-to-car.org.

[2] “The Dedicated Short Range Comms Working Group.” http://grouper.iee.org/groups/scc32/dsrc/index.html.

[3] “The IEEE802.11p Task Group.” http://grouper.ieee.org/groups/802/11/Reports/tgp_update.htm.

[4] Vehicle Safety Communications Consortium, "Vehicle safety communications project task 3 final report: Identify intelligent vehicle safety applications enabled by DSRC," tech. rep., National Highway Traffic Safety Administration, March 2005.

[5] “Networks on Wheels - NOW." http://www.network-on-wheels.de. 
[6] “The PReVENT Project.” http://www.prevent-ip.org/.

[7] “Intelligent Transportation Systems, U.S. Department of Transportation.” http://www.its.dot.gov.

[8] U. Lee, B. Zhou, M. Gerla, E. Magistretti, P. Bellavista, and A. Corradi, "Mobeyes: Smart mobs for urban monitoring with a vehicular sensor network," IEEE Wireless Communications, vol. 13, no. 5, pp. 52-57, 2006.

[9] U. Lee, J.-S. Park, E. Amir, and M. Gerla, "FleaNet: A virtual market place on vehicular networks," in In Proc. of Mobiquitous'06, (San Jose, CA, USA), July 2006.

[10] M. Caliskan, D. Graupner, and M. Mauve, "Decentralized discovery of free parking places," in VANET'06, (Los Angeles, CA, USA), pp. 30-39, Sept. 2006.

[11] T. Lomax and D. Schrank, "Urban mobility study," tech. rep., Texas Transportation Institute, 2005.

[12] M. Shinkman and M. Buchanan, "Driving change: How policymakers are using road charging to tackle congestion," tech. rep., Economist Intelligence Unit, Nov. 2006.

[13] S. Dashtinezhad, T. Nadeem, B. Dorohonceanu, C. Borcea, P. Kang, and L. Iftode, "TrafficView: A driver assistant device for traffic monitoring based on car-to-car communication,” IEEE Vehicular Technology Conference, 2004.

[14] S. Dornbush and A. Joshi, "StreetSmart traffic: Discovering and disseminating automobile congestion using VANET's," in IEEE Vehicular Technology Conference, (Dublin, Ireland), April 2007.

[15] L. Wischof, A. Ebner, and H. Rohling, "Information dissemination in self organising intervehicle networks," IEEE Transactions on Intelligent Transportation Systems, vol. 6, pp. 90-101, March 2005.

[16] U. Lee, Magistretti, B. Zhou, M. Gerla, B. Paola, and A. Corradi, "Efficient data harvesting mobile sensor platforms," in IEEE Conference on Pervasive Computing, (Pisa, Italy), 2006.

[17] M. Torrent-Moreno, A. Festag, and H. Hartenstein, "System design for information dissemination in VANETs," in 3rd International Workshop on Intelligent Transportation, (Hamburg, Germany), March 2006.

[18] G. Seetharaman, A. Lakhotia, and E. P. Blasch, "Unmanned vehicles come of age: The DARPA grand challenge," IEEE Computer Magazine, vol. 39, pp. 26-29, Dec. 2006.

[19] M. Bertozzi, A. Broggi, and A. Fascioli, "VisLab and the evolution of vision-based UGVs," IEEE Computer Magazine, vol. 39, pp. 31-38, Dec. 2006.

[20] B. Touchton, T. Galluzzo, D. Kent, and C. Crane, "Perception and planning architecture for autonomous ground vehicles,” IEEE Computer Magazine, vol. 39, pp. 40-47, Dec. 2006.

[21] J. Connors and G. Elkaim, "Analysis of a spline based, obstacle avoiding path planning algorithm," in IEEE Vehicular Technology Conference, (Dublin, Ireland), April 2007.

[22] V. Gradinescu, C. Gorgorin, R. Diaconescu, V. Cristea, and L. Iftode, "Adaptive traffic lights using car-to-car communication,” in IEEE Vehicular Technology Conference, (Dublin, Ireland), April 2007.

[23] M. Hartong, R. Goel, C. Farkas, and D. Wijesekera, "PTC-VANET interactions to prevent highway rail intersection crossing accidents," in IEEE Vehicular Technology Conference, (Dublin, Ireland), April 2007.

[24] C. C. C. UK, “The 2006 traffic monitoring report,” tech. rep., Cambridgeshire County Council, 2006.

[25] S. Youssef, Iterative Computer Algorithms with Applications in Engineering: Solving Combinatorial Optimization Problems, ch. 3, pp. 109-181. IEEE Computer Society, 1999.

[26] R. Barr, Z. J. Haas, and R. van Renesse, "JiST: An efficient approach to simulation using virtual machines.," Software Practice \& Experience, vol. 35, pp. 5539-576, May 2005.

[27] R. Barr, Z. J. Haas, and R. van Renesse, Handbook on Theoretical and Algorithmic Aspects of Sensor, Ad hoc Wireless, and Peer-to-Peer Networks., ch. 19: Scalable Wireless Ad hoc Network Simulation, pp. 297-311. CRC Press, 2005.

[28] D. R. Choffnes and F. E. Bustamante, "STRAW - an integrated mobility and traffic model for VANETs," in International Command and Control Research and Technology Symposium (CCRTS), (McLean, VA, USA), June 2005.

[29] B. Karp and H. Kung, "GPSR: Greedy perimeter stateless routing for wireless networks,” MOBICOM 2000, pp. 243 $-254,2000$. 